\title{
A NOTE ON EVALUATION OF THE INTEGRAL
}

$$
\int_{0}^{\infty} e^{-k t} I_{0}^{n}(t) d t
$$

by R. P. AGARWAL

(Received 10th October 1963)

1. The object of this note is to show that the integral

$$
\int_{0}^{\infty} e^{-k t} I_{0}^{n}(t) d t
$$

for certain particular values of $k$ and $n$ can be evaluated in terms of complete elliptic integrals. The integral (1.1) for $n=1$ can be easily expressed as a binomial and for $n=2$ in terms of a complete elliptic integral (2). However, the corresponding value for other cases does not appear to be given in the literature.

In this note, I use an indirect method to evaluate (1.1) for $k=3=n$, in terms of a square of a complete elliptic integral. In the sequel, an interesting case of reducibility of a particular $F_{c}$-function (one of the Lauricella's hypergeometric functions of three variables (1)) is obtained.

\section{Evaluation of (1.1) for $k=3=n$}

It is very easy to see that (1.1) can be evaluated for positive integral values of $n$ by term by term integration of the $n$-ple series representing $I_{0}^{n}(t)$. In fact, simple algebra shows that

$$
\int_{0}^{\infty} e^{-k t} I_{0}^{n}(t) d t=\frac{1}{k} F_{c}\left(\frac{1}{2}, 1 ; 1, \ldots, 1 ; \frac{1}{k^{2}}, \ldots, \frac{1}{k^{2}}\right),
$$

where $F_{c}$ is one of the four Lauricella's hypergeometric functions of $n$-variables.

For $k=3=n,(2.1)$ gives

$$
\int_{0}^{\infty} e^{-3 t} I_{0}^{3}(t) d t=\frac{1}{3} F_{c}\left(\frac{1}{2}, 1 ; 1,1,1 ; \frac{1}{9}, \frac{1}{9}, \frac{1}{9}\right) .
$$

Now, since

$$
I_{0}(t)=\frac{1}{\pi} \int_{0}^{\pi} e^{t \cos u} d u
$$


the left hand side of (2.2) can be written as

$$
\frac{1}{\pi^{3}} \int_{0}^{\infty} e^{-3 t}\left[\int_{0}^{\pi} \int_{0}^{\pi} \int_{0}^{\pi} e^{t(\cos u+\cos v+\cos w)} d u d v d w\right] d t
$$

Interchanging the order of integration, which is obviously justified, this becomes

$$
\frac{1}{\pi^{3}} \int_{0}^{\pi} \int_{0}^{\pi} \int_{0}^{\pi} \frac{d u d v d w}{3-\cos u-\cos v-\cos w}
$$

Evaluating (2.3) by the help of a known integral due to Watson (3), we have

$$
\int_{0}^{\infty} e^{-3 t} I_{0}^{3}(t) d t=(18+12 \sqrt{2}-10 \sqrt{3}-7 \sqrt{6})\left(\frac{2 K_{2}}{\pi}\right)^{2},
$$

where $K_{2}$ is the complete elliptic integral with modulus $(2-\sqrt{3})(\sqrt{3}-\sqrt{2})$.

(2.4) gives the desired result.

Incidentally, we have shown that

$$
\frac{1}{3} F_{c}\left(\frac{1}{2}, 1 ; 1,1,1 ; \frac{1}{9}, \frac{1}{9}, \frac{1}{9}\right)=(18+12 \sqrt{2}-10 \sqrt{3}-7 \sqrt{6})\left(\frac{2 K_{2}}{\pi}\right)^{2}
$$

It does not appear to be easy to establish the reduction in (2.5) by direct transformation of its left hand series.

\section{REFERENCES}

(1) P. APpell and J. KAMPE DE FerIet, Fonctions Hypergeometriques et Hyperspheriques (Paris, 1926).

(2) A. ERdéLyi et al., Tables of Integral Transform, Vol. 1 (Bateman Manuscript Project).

(3) G. N. WAtSon, Quart. Jour. Math. (Oxford), 10 (1939), 266-276.

\section{DePaRTMENT OF MATHEMATICS}

UNIVERSITY OF GORAKHPUR

GORAKHPUR 